\title{
Politische Partizipation in der UdSSR als Forschungsproblem im Kalten Krieg - Eine historische Rückschau ${ }^{1}$
}

Frithjof Benjamin Schenk

\section{Einleitung}

Die Auseinandersetzung mit Fragen politischer Partizipation findet traditionellerweise vor allem in der politikwissenschaftlichen Forschung statt. Rainer-Olaf Schultze hat „Partizipation“ gar als „Schlüsselbegriff politikwissenschaftlicher Theorie und politischer Praxis“ bezeichnet. ${ }^{2}$ Als „Partizipation“ gelten nach seiner Definition ,alle diejenigen Formen politischer Beteiligung, die Bürger freiwillig, individuell und/oder kollektiv im Verbund mit anderen unternehmen, um politische Entscheidungen direkt oder indirekt zu ihren Gunsten zu beeinflussen. "3 Als gegenwartsorientierte Sozialwissenschaft befasst sich die Partizipationsforschung vor allem mit Fragen der Teilnahme bzw. Teilhabe von Bürgern am politischen Prozess in modernen Demokratien. Zunächst interessierte sie sich dabei vor allem für jene Möglichkeiten des Einzelnen, in formalen und legalen Strukturen (z. B. im Rahmen von Wahlsystemen, Wahlkämpfen und Parteien) an politischen Entscheidungsprozessen und Repräsentationsvorgängen mitzuwirken, wobei zwischen Formen der „direkten“ und ,indirekten“ bzw. der ,konventionellen“ und „unkonventionellen Partizipation“" unterschieden wurde. ${ }^{4}$ Nicht zuletzt vor dem Hintergrund der Studentenproteste der 1960er Jahre und der damit verbundenen „partizipatorischen Revolution“ erfuhr das Konzept der Partizipation in der Forschung eine deutliche Erweiterung. ${ }^{5}$ Seither werden auch unkonventionelle Formen politischen Handelns, wie zum Beispiel

1 Ich danke Petra Stykow und Bianca Hoenig für Literaturhinweise und konstruktive Kritik an früheren Versionen dieses Textes.

2 Schultze 1995, 396.

3 Schultze 2005, 675. Zu einer älteren Definition vgl. Verba u. a. 1978, referiert u. a. bei Hahn 1988, 27.

4 Vgl. Brockhaus Enzyklopädie 2006, 65.

5 Schultze 1995, 404. 
Bürgerproteste und Bürgerinitiativen sowie wilde Streiks, die Verweigerung formaler Mitwirkung (z. B. Wahlboykott) oder die Ausübung politisch motivierter Gewalt als Formen politischer Partizipation diskutiert. ${ }^{6}$ Heute differenziert die politikwissenschaftliche Forschung zwischen einem ,instrumentellen“ und einem ,normativen“ Verständnis von Partizipation sowie zwischen unterschiedlichen Formen der politischen Teilhabe, die von der Teilnahme an Wahlen bis zur Ausübung von Gewalt gegen Personen und Sachen reichen. ${ }^{7}$

Anders als in der Politikwissenschaft hat das Konzept der Partizipation in der Geschichtswissenschaft (noch) keine vergleichbare forschungsleitende Kraft entfalten können. Zwar interessieren sich auch Historiker für Fragen der Teilnahme und Teilhabe von Menschen am politischen Prozess in unterschiedlichen Epochen. Fragen wie diese werden in der historischen Forschung jedoch eher unter anderen Begriffen verhandelt, wie zum Beispiel dem der „Loyalität“", der „Treue“ oder der „Öffentlichkeit “ .8 Da sich die Geschichtswissenschaft in den 1960er und 1970er Jahren bei der Suche nach theoretischen Modellen und Konzepten häufig an den Sozialwissenschaften wie der Soziologie oder der Ökonomie orientierte, wäre es eine interessante Frage, warum es das Konzept der Partizipation nicht geschafft hat, die historische Forschung nachhaltig zu beeinflussen. Dass der Begriff der Partizipation seit jeher einen stark wertenden Beiklang hat, kann dabei kaum als Erklärungsgrund gelten. Schließlich haben es auch andere normativ geladene Theorien und Konzepte, wie zum Beispiel jenes der „Modernisierung" geschafft, die geschichtswissenschaftlichen Debatten seit den 1960er Jahren maßgeblich zu beeinflussen. ${ }^{9}$

Während sich die heutige vergleichende Partizipationsforschung in erster Linie mit Formen politischer Teilhabe in demokratisch verfassten Staaten befasst, war diese Forschungsrichtung in den 1970er und 1980er Jahren ein integraler Bestandteil des politischen Systemvergleichs. Seit Mitte der 1970er Jahre beschäftigte sich vor allem die US-amerikanische Kommunismus- und Sowjetunion-Forschung intensiv mit der Frage, in welchem Maße auch kommunistisch verfasste Systeme ihren Bürgern Spielräume politischer Partizipation eröffneten, bzw. in welchem Maße

6 Brockhaus Enzyklopädie 2006, 66.

7 Vgl. dazu u. a. Schultze 2005, 676. Einen guten Überblick bietet auch: Kaase 1995.

8 Vgl. u. a. Zimmermann 2010; Buschmann/Murr 2008; Rittersporn u. a. 2003.

9 Vgl. Wehler 1975. Kritisch dazu u. a. Mergel 1997. 
man hier überhaupt von einer „Teilhabe“ an den politischen Entscheidungsprozessen sprechen konnte.

Der Boom der Partizipationsforschung, der auch die westliche Osteuropa-Kunde in diesen Jahren erfasste, hatte zum einen mit der bereits angesprochenen allgemeinen Sensibilisierung für das Thema in den 1960er Jahren zu tun. Zum anderen reflektierte dieser Trend jedoch auch einen von außen deutlich wahrnehmbaren politischen Wandel innerhalb des politischen Systems der UdSSR nach dem Tod Stalins und dem 20. Parteitag der KPdSU (1956), der unter anderem in der Verabschiedung eines neuen Parteiprogramms im Jahr 1961 und der Ausarbeitung einer neuen sowjetischen Verfassung im Jahr 1977 zum Ausdruck kam. Für westliche Forscher stellte sich nun verstärkt die Frage, inwiefern sich mit diesen Reformen auch neue Möglichkeiten der Teilhabe der sowjetischen Bürger am politischen Prozess eröffneten.

Im Folgenden soll der Blick auf die Debatten dieser systemvergleichenden westlichen Partizipationsforschung der 1970er sowie 1980er Jahre gerichtet und deren Erkenntnisinteresse, Hypothesen und zum Teil kontroverse Forschungsergebnisse beleuchtet werden. Ziel ist es nicht, die damals diskutierte Frage zu beantworten, ob, bzw. in welchem Maße, es politische Partizipation in der UdSSR in den 1970er und 1980er Jahren tatsächlich gegeben hat oder nicht. Vielmehr wird aus wissenschaftshistorischer Perspektive danach gefragt, welche Rolle das Konzept der (politischen) „Partizipation“ in westlichen Analysen des politischen Systems der UdSSR in den letzten Jahrzehnten des Kalten Krieges spielte und inwieweit dieses zum Verständnis der politischen Entwicklung jenseits des „Eisernen Vorhangs" beigetragen hat.

Dabei wird die These vertreten, dass es der westlichen Sowjetologie der 1970er und 1980er Jahre dank des Konzepts der „Partizipation“ zwar gelang, überkommene und starre Vorstellungen der TotalitarismusTheorie aufzuweichen und den Blick für Prozesse des politischen Wandels in der UdSSR zu schärfen. Durch ihre Fokussierung auf konventionelle und legale Formen politischer Teilhabe blieb die Forschung jedoch auf das bestehende politische System fixiert, dessen Wandel sie zwar für möglich hielt, an dessen Fortbestehen sie jedoch genauso fest glaubte wie das Politbüro in Moskau. Aufgrund dieser Konzentration auf systemkonforme Optionen politischer Partizipation (Mitgliedschaft in der KPdSU oder im sowjetischen Jugendverband (Komsomol), Teilnahme an Wahlen, Engagement in politischen Ämtern, Schreiben von Eingaben an Volksvertreter und Beamte etc.) blieb die westliche Osteuropa-Forschung blind 
für jene politischen Bewegungen im Untergrund, von denen wir heute annehmen, dass sie eine wichtige Rolle bei der Erosion des sowjetischen Politik-Systems gespielt haben (nationale Bewegungen, Dissidentenbewegung, non-konforme Jugendkultur, Umweltbewegungen etc. $)^{10}$

Die Fixierung der westlichen Sowjetologie auf die offizielle ,,politische Kultur" der UdSSR und die Nicht-Berücksichtigung ,außer-systemischer" Formen des politischen und sozialen Lebens hatte zum einen damit zu tun, dass ausländische Forscher bis in die 1980er Jahre daran glaubten, die Struktur und das Funktionieren des sowjetischen Systems ließen sich mit Blick auf dessen ideologische Fundierungen (Schriften von Marx, Lenin etc.) verstehen und erklären. Zum anderen kann das Unvermögen der westlichen UdSSR-Experten, tatsächliche politische Prozesse innerhalb des „Ostblocks“ zu erfassen, auch damit erklärt werden, dass sie versuchten, ihren Untersuchungsgegenstand mithilfe theoretischer Konzepte zu deuten, die der Analyse westlicher Gesellschaften entlehnt waren. Auch im Falle der Partizipationsforschung kamen Sozialwissenschaftler vielfach nicht weiter als bis zur Diagnose von Defiziten der sowjetischen Gesellschaft, d. h. zur Feststellung, dass man in der UdSSR bestimmte Formen der politischen Teilhabe, die man aus dem Westen kannte, eben nicht finden könne. Von einem Verständnis jener politischen und sozialen Prozesse, die in der UdSSR tatsächlich abliefen, war man dabei weit entfernt. Dessen wurde man sich bei allen westlichen Think tanks spätestens im August 1991 bewusst, als die Sowjetunion - für fast alle Beobachter unerwartet - von einem Tag auf den anderen aufhörte zu existieren. ${ }^{11}$

\section{Partizipation als Forschungsgegenstand westlicher Kommunismus- und Sowjetunion-Forschung der 1970er und 80er Jahre}

1981 bezeichnete der amerikanische Politologe Donald Schulz das Thema „Politische Partizipation “als ,one of the most fashionable in the field of comparati-

10 Über die Gründe des Systemwechsels in Osteuropa in den Jahren 1985-1991 wird bis heute kontrovers diskutiert. Vgl. exemplarisch: Karklins 1994; Beissinger 2009 (mit weiteren Literaturangaben); Yurchak 2006. Zur Diskussion der frühen 1990er Jahre vgl. z. B. v. Beyme 1994, 46-61.

11 Zur Kritik an der westlichen Osteuropaforschung des Kalten Krieges: Simon 1992; Riescher/Gabriel 1993, 7-16; v. Beyme 1994, 16-34; Manteufel u. a. 2000. 
ve communism“" ${ }^{12}$ Tatsächlich lässt sich vor allem in der US-amerikanischen Kommunismus- und Sowjetunionforschung seit Mitte der 1970er Jahre eine wahre ,participation explosion“ 13 beobachten. Dieses verstärkte Interesse der westlichen Forschung an Fragen der politischen Teilhabe in Ländern des „Ostblocks“ lässt sich einerseits mit dem Ruf nach ,,mehr Partizipation“ in Verbindung bringen, der sich im Westen in den Studentenbewegungen der 1960er Jahre Gehör verschaffte. ${ }^{14}$ Folgt man der Diagnose von Carole Pateman, so erfuhr das Konzept der Partizipation in dieser Zeit eine signifikante Um- und Aufwertung. Während man Mitte des 20. Jahrhunderts in den westlichen Demokratien Forderungen nach mehr Partizipation der Bürger noch mit Zurückhaltung begegnete, betrachtete man ein höheres $\mathrm{Maß}$ an politischer Teilhabe nun als ein anzustrebendes Ziel. ${ }^{15}$ Andererseits wurden amerikanische Sowjetunion-Forscher auch durch Prozesse des politischen Wandels innerhalb der UdSSR mit der Frage nach neuen Spielräumen politischer Teilhabe jenseits des Eisernen Vorhangs konfrontiert. Mit Erstaunen nahmen zum Beispiel westliche Politologen den überproportionalen Anstieg von Mitgliederzahlen der KPdSU, des Komsomol und anderer gesellschaftlicher Organisationen seit den späten 1950er Jahren zur Kenntnis. ${ }^{16}$ Registriert wurde zudem, dass auch auf der ideologischen und verfassungsrechtlichen Ebene (zumindest formal) die Weichen für eine größere Bürgerbeteiligung an politischen Entscheidungsprozessen (vor allem auf der lokalen Ebene) gestellt wurden. Insbesondere die Neubelebung der Räte (Sowjets) auf lokaler Ebene, von der sich die sowjetische Regierung offenbar eine stärkere Integration der Bevölkerung in Prozesse der lokalen Administration versprach, wurde von amerikanischen Politologen als Zeichen einer ,neuen Zeit“" gewertet, deren Bedeutung man nun nachspüren wollte. ${ }^{17}$

In dieser Situation stellte die westliche Partizipationsforschung der 1970er Jahre wichtige Grundannahmen der bisher vorherrschenden Totalitarismus-Theorie auf den Prüfstand. Vertreter dieser Schule hatten argu-

12 Schulz 1981, 1.

13 Schulz 1981, 2.

14 Vgl. Pateman 1970, 1.

15 In Misskredit war das Konzept der Partizipation zeitweise deshalb gekommen, weil sich sowohl Nationalsozialismus als auch Stalinismus dezidiert auf die Mobilisierung der „Massen“ (mass participation) gestützt hatten, wenngleich diese aus westlicher Sicht durch erzwungene ,mobilization“, d. h. durch Druck und Einschüchterung durch das Regime zustande gekommen war.

16 Vgl. Hough 1976, 7ff.

17 Dies gilt insbesondere für die Pionierstudie von Friedgut 1979. 
mentiert, dass jede Form politischer Teilhabe in einem kommunistischen Land als das Ergebnis einer erzwungenen „Mobilisierung“ der Bevölkerung durch die Regierung anzusehen sei. ${ }^{18}$ Die hohen Mitgliederzahlen der KPdSU und des Komsomol, die Beteiligung der Menschen an Aufmärschen, Kundgebungen und gemeinschaftlicher Arbeit, die Mitarbeit von Millionen Menschen in den Räten (Sowjets) auf verschiedenen Verwaltungsebenen, die hohe Wahlbeteiligung - all das wurde auf Zwang und Nötigung der Bevölkerung durch ein von einer kleinen Parteielite getragenes Regime zurückgeführt. Die Partizipationsforschung gab sich mit dieser Diagnose nicht zufrieden. Ähnlich wie in der Geschichtswissenschaft, wo sich eine junge Garde sogenannter „Revisionisten“ daran machte, das ideologische Gebäude der Totalitarismus-Forschung und deren Bild des sowjetischen Machtsystems (insbesondere des Stalinismus) zu demontieren, verstärkten sich in den 1970er Jahren auch unter westlichen Politologen Zweifel, ob das sowjetische System wirklich allein auf Unterdrückung und „mobilisierter Beteiligung “ basiere. ${ }^{19}$ Die Tatsache, dass die Mitgliederzahlen der KPdSU nach dem Ende der Schreckensherrschaft Stalins nicht nach unten, sondern deutlich nach oben gingen, ließ Zweifel an der vereinfachten Sicht der Totalitarismus-Forschung aufkommen.

Wenngleich sich westliche Politologen, die sich in den 1970er Jahren mit Fragen der politischen Partizipation in der UdSSR befassten, deutlich von der Totalitarismus-Forschung abgrenzten, blieben die meisten doch in den binären Denkschemata des Kalten Krieges verhaftet. Unhinterfragt blieb beispielsweise die Annahme, dass sich „westliche Demokratien“ und die kommunistischen Regimes jenseits des Eisernen Vorhangs hinsichtlich der ,,politischen Kultur" deutlich voneinander unterschieden. ${ }^{20}$ Auch die Partizipationsforschung war darum bemüht, die Besonderheiten dieser fremden politischen Kultur der sozialistischen Welt zu ergründen bzw. besser zu verstehen. ${ }^{21}$ Allerdings gingen sie - stärker als die Vertreter der Totalitarismus-Theorie - von der Möglichkeit des Wandels der politischen Kultur innerhalb des „Ostblocks“ aus. ${ }^{22}$

18 Vgl. z. B. den ein „Klassiker“ der Totalitarismus-Theorie der 1950er Jahre: Friedrich/Brzezinski 1956.

19 Zur Schule der Revisionisten in der westlichen Historiographie vgl. u. a. Cohen 1985, 3-37; Fitzpatrick 1992; Hildermeier 1997; Plaggenborg 1998.

20 Vgl. z. B. DiFranceisco/Gitelman 1984. Kritisch dazu Cohen 1985, 32.

21 Vgl. exemplarisch: White 1977; White 1979. Kritisch dazu: v. Beyme 1994, 328-333.

22 Cohen 1985, 30. 
Mit dieser auf das bestehende System fixierten Sichtweise ging einher, dass die westliche Partizipationsforschung dem institutionellen Rahmen, in dem Bürger sozialistischer Staaten an politischen Prozessen teilhaben konnten, großes Interesse entgegenbrachten und intensiv nach den ideologischen Grundlagen fragten, auf denen die Idee der Partizipation in Gesellschaften sowjetischen Typs basierte. ${ }^{23}$ In diesem Zusammenhang spielte die Analyse von zwei Schlüsseltexten eine besondere Rolle: Zum einen die Schrift Der Bürgerkrieg in Frankreich von Karl Marx aus dem Jahr 1871, ${ }^{24}$ zum anderen das Traktat Staat und Revolution. Die Lehre des Marxismus vom Staat und die Aufgaben des Proletariats in der Revolution von Vladimir Il'ič Lenin, geschrieben im August/September $1917 .^{25}$

\section{II.1 Marx und Lenin zur Frage der politischen Partizipation}

Die Pariser Kommune war ein wichtiger historischer Referenzpunkt Lenins und der Bol'seviki, wenn es um die Frage ging, wie der ,ideale Staat“ nach der angestrebten Revolution in Russland zu organisieren sei. ${ }^{26}$ Lenin bezog sich in seinen sogenannten Aprilthesen aus dem Jahr 1917 explizit auf das französische Vorbild von 1871 und ging dabei ausführlich auf die Darstellung der Pariser Kommune bei Karl Marx ein. ${ }^{27}$ In seiner Darstellung Der Bürgerkrieg in Frankreich hatte Marx im Sommer 1871 ein detailliertes

23 Beide Schriften werden eingehend referiert bei: Friedgut 1979, 32-41 und Hahn 1988, 59-61.

24 Marx 1871/1973.

25 Lenin 1917/1960.

26 Friedgut 1979, 35.

27 Lenin 1917/1959 - Lenin fordert hier nicht die Schaffung einer ,parlamentarische[n] Republik - eine Rückkehr von den Arbeiterdeputiertenräten zu dieser wäre ein Schritt rückwärts -, sondern eine Republik von Arbeiter-, Landarbeiterund Bauerndeputiertenräten im ganzen Lande, von unten bis oben. [Die] Abschaffung der Polizei, der Armee und des Beamtentums. [Die] Entlohnung aller Beamten, die durchweg wählbar und jederzeit absetzbar sein müssen, nicht über dem Durchschnittslohn eines qualifizierten Arbeiters.“ (These Nr. 5). In das Parteiprogramm der „Kommunistischen Partei" sollte die „Forderung eines ,Kommunestaates', d.h. eines Staates nach dem Vorbild der Pariser Kommune aufgenommen werden (These 9.b.2.). Dabei solle man sich daran erinnern, wie „Marx und Engels in den Jahren 1871, 1872 und 1875 über die Erfahrung der Pariser Kommune urteilten, und darüber, was für einen Staat das Proletariat braucht." Ebd. $5 f$. 
Bild von der Organisation der Commune gezeichnet. ${ }^{28}$ Die Partizipationsforschung interessierte besonders, wie Marx in diesem Portrait einer vergangenen, idealen politischen Ordnung das Verhältnis von städtischer Bevölkerung, Polizei, Heer, Gerichtswesen, Regierung, Administration und Parlament (bzw. Volksvertretung) beschrieb.

Marx betont, die Pariser Kommune habe sich aus „durch allgemeines Stimmrecht in den verschiedenen Bezirken von Paris gewählten Stadträten" gebildet. ${ }^{29}$ Diese Räte, die sich mehrheitlich aus der Arbeiterschaft rekrutierten (oder ,anerkannte Vertreter der Arbeiterklasse“ waren), seien ihren Wählern gegenüber „verantwortlich und jederzeit absetzbar" gewesen. Die Versammlung oder Vereinigung der gewählten Räte sei keine ,parlamentarische, sondern eine arbeitende Körperschaft" gewesen, die sowohl legislative als auch exekutive Funktion innehatte. ${ }^{30}$ Marx hebt hervor, dass die Mitglieder der Commune, wie auch alle anderen Beschäftigten des öffentlichen Dienstes, lediglich „Arbeiterlohn“ erhielten. Die Polizei und das stehende Heer, die Marx als „Werkzenge der materiellen Macht der alten Regierung“ bezeichnet, seien dagegen in ihrer bisherigen Form abgeschafft worden. Die Kommunarden hätten die Polizei in das ,verantwortliche und jederzeit absetzbare Werkzeng der Kommune" verwandelt, die Funktionen des Heeres wollte man auf dem Land einer Volksmiliz übertragen. Auch die „richterlichen Beamten" sollten in diesem idealen Gemeinwesen ,gewählt, verantwortlich und absetzbar sein." 31

Marx zufolge verfolgten die Kommunarden von Paris das Ziel, ein System der „Selbstregierung der Produzenten“ (d. h. des Proletariats) in ganz Frankreich zu etablieren. ${ }^{32}$ In allen größeren städtischen Zentren des Landes sollte die „kommunale Ordnung der Dinge“ nach Pariser Vorbild eingeführt werden. Einer ,Skizze der nationalen Organisation“ zufolge habe man angestrebt, die Kommune als ,politische Form“ auch noch im kleinsten Dorf durchzusetzen. Alle Landgemeinden bzw. Landkommunen eines Bezirks sollten ihre gemeinsamen Angelegenheiten durch eine „Versammlung von Abgeordneten " in der Bezirkshauptstadt verwalten, die wiederum Abgeordnete zur „Nationaldelegation“ in Paris entsenden sollten. Auch die Mitglieder der Bezirks- bzw. der Nationaldelegationen sollten jederzeit absetz-

28 Marx 1871/1973, insbes. 336-340. Zur Analyse der Pariser Kommune bei Karl Marx vgl. auch: Meschkat 1971, 1075-1079.

29 Marx 1871/1973, 339.

30 Marx 1871/1973, 339.

31 Marx 1871/1973, 339.

32 Marx 1871/1973, 339. 
bar und an die ,bestimmten Instruktionen ihrer Wähler gebunden sein“. ${ }^{33}$ Alle „gemeinsamen Angelegenheiten“ sollten, so liest Marx die genannte Skizze der nationalen Organisation, auf lokaler bzw. Bezirksebene geregelt werden. Die wenigen verbleibenden Funktionen, die in die Zuständigkeit einer Zentralregierung fielen, wollten die Kommunarden ,streng verantwortliche[n] Beamte $[n]$ “ der Kommune übertragen. Die „Staatsmacht" in ihrer bisherigen Form wollten die Revolutionäre (aus der Sicht von Marx) ,vernichten“. Die „Einheit der Nation“ sollte nur noch durch die „Kommunalverfassung“ organisiert werden. ${ }^{34}$

Fasst man die Besonderheiten dieser Kommunalverfassung in der Interpretation von Marx noch einmal zusammen, so lassen sich als wichtigste Charakteristika das , allgemeine Stimmrecht des in Kommunen konstituierten Volkes", die direkte und gleiche Wahl der Räte und richterlichen Beamten sowie die „Verantwortlichkeit" und „Absetzbarkeit“ der Räte, Richter, Abgeordneten und Beamten hervorheben. Marx skizziert hier das Bild eines idealen Gemeinwesens, in dem die Macht der alten sozialen (,,besitzenden“) Klassen gebrochen und durch ein „System der Selbstregierung“ der Arbeiterklasse ersetzt werden sollte.

Marx' Analyse der Kommunalverfassung von Paris aus dem Jahr 1871 war für die amerikanische Partizipationsforschung der 1970er und 1980er Jahre vor allem deshalb von Interesse, weil sich Lenin in seinen Aprilthesen und später in seinem Werk Staat und Revolution explizit auf das hier vorgestellte Modell des kommunalen Staates bezog. Folgt man Lenins Ausführungen in diesen beiden Traktaten (wie es die Partizipationsforscher taten), so schwebte dem Revolutionsführer in Russland der Aufbau eines Gemeinwesens nach dem von Marx entworfenen Bild der Pariser Kommune vor. Lenin verfasste sein Buch Staat und Revolution im Sommer 1917, d. h. noch vor dem coup d'état der Bol'ševiki im Oktober (November) desselben Jahres. Der Text ist somit als eine theoretische Reflexion über die neu zu errichtende soziale Ordnung nach dem Sturz des ancien régime in Russland zu lesen.

In seinem Werk widmet Lenin ein ganzes Kapitel der Analyse von Marx' Schrift zur Pariser Kommune. ${ }^{35}$ Dabei schließt er sich dessen Forde-

33 Marx 1871/1973, 340.

34 Marx 1871/1973, 340.

35 Lenin 1917/1960, 426-445. Zum Wandel der Beurteilung der Pariser Kommune bei Lenin: Meschkat 1971, 1082-1085. Meschkat betont, dass Lenin 1905 die führende Rolle der Arbeiterklasse in der Pariser Erhebung noch bestritten habe. In der Schrift Zwei Taktiken der Sozialdemokratie in der demokratischen Revolution habe 
rung an, dass die Arbeiterklasse in bzw. nach einer Revolution nicht Besitz von der bisherigen „Staatsmaschine“ ergreifen solle. Vielmehr gelte es, die alten politischen Machtstrukturen zu ,zerschlagen“" und zu ,zerbrechen“. 36 Ausführlich zitiert Lenin Marx' Schrift Die Revolution in Frankreich, darunter auch die oben erwähnten Passagen, in denen von der inneren Organisation der Pariser Kommune die Rede ist: Die „Beseitigung des stehenden Heeres [und die] vollkommene Wählbarkeit und Absetzbarkeit aller Amtspersonen“ interpretiert Lenin als Ausdruck einer „vollständigeren Demokratie“. In Paris habe sich 1871 die ,bürgerliche Demokratie“ in eine „proletarische Demokratie“ verwandelt, der Staat (als ,,besondere Gewalt zur Unterdrückung einer bestimmten Klasse“) in etwas, ,was eigentlich kein Staat mehr ist. " ${ }^{37}$

Lenin zufolge war die Kommune daran gescheitert, dass die Kommunarden von Paris den Widerstand der Bourgeoisie ,nicht entschlossen genug" niedergehalten hätten. Diesen Fehler dürfe man in Zukunft nicht wiederholen. Offen predigt Lenin die „Diktatur des Proletariats“, die in einer Übergangsphase zwischen Kapitalismus und Kommunismus unerlässlich sei. Rechtfertigen ließe sich diese vorübergehende Unterdrückung der ehemaligen „Bedrücker" damit, dass das unterdrückende Organ (d.h. die Machtinstrumente der Arbeiter und Bauern) ,schon die Mehrheit ist" und nicht mehr die „Minderheit der Bevölkerung“. ${ }^{38}$ Tatsächlich wird hier Partizipation als „,demokratische“ Teilhabe an der Unterdrückung der besitzenden Klassen propagiert. In einem solcherart strukturierten Gemeinwesen werde es, so Lenin, zum „Absterben des Staates“ in seiner bisherigen Form kommen, wie es Engels in seiner Schrift „Anti-Dühring“ beschrieben hatte. ${ }^{39}$

Lenin sah in der Pariser Kommune die Überwindung der ,bürgerlichen“ durch die ,proletarische Demokratie“ in einem erheblichem Maße verwirklicht. Für Lenin stellte sich dieser Prozess als Übergang von der „Unterdrückerdemokratie“ zur „Demokratie der unterdrückten Klassen“, vom

er polemisiert, dass die Pariser „Arbeiterregierung [...] die Aufgaben des Kampfes für die Republik und die Aufgaben des Kampfes für den Sozialismus verwechselt [habe]" und sie nicht imstande gewesen sei, ,die Aufgaben einer energischen militärischen Offensive gegen Versailles zu lösen“, wie z. B. sich der Bank von Frankreich zu bemächtigen. Zit. nach Meschkat 1971, 1082f. Zu Lenins Marx-Lektüre vgl. auch Hahn 1988, $59 f$.

36 Lenin 1917/1960, 427, 429 (Kursiv im Original bei Marx, übernommen von Lenin).

37 Lenin 1917/1960, 432.

38 Lenin 1917/1960, 432.

39 Zit. bei Lenin 1917/1960, 407f. bezugnehmend auf Engels 1878/1953. 
„Staat als ,besonderer Gewalt' zur Niederhaltung einer bestimmten Klasse, [hin] zur Niederhaltung der Unterdrücker durch die allgemeine Gewalt der Mehrheit des Volkes, der Arbeiter und Bauern“ dar. ${ }^{40}$ Die ,uneingeschränkte Wählbarkeit und die jederzeitige Absetzbarkeit ausnahmslos aller beamteten Personen" - wichtige Charakteristika der Pariser Kommune in der Analyse von Marx - bezeichnet Lenin als ,einfache und ,selbstverständliche“ demokratische Maßnahmen“". 41 Gleichzeitig betont er, dass nur mit diesen Mitteln, die allein die ,politische Umgestaltung der Gesellschaft" betreffen, der Schritt vom Kapitalismus zum Sozialismus vollzogen werden könne. ,[V]ollen Sinn und Bedeutung“" würden sie jedoch erst im Zusammenhang mit einer umfassenden Enteignung der „Expropriateure“ entfalten, d.h. ,,mit dem Übergang des kapitalistischen Privateigentums an den Produktionsmitteln in gesellschaftliches Eigentum." ${ }^{\text {"42 In }}$ diesem Punkt wollte Lenin deutlich aus den ,Fehlern“ der Pariser Kommune lernen und das Rad der Revolution beim nächsten Anlauf deutlich weiter drehen.

Keinen Zweifel ließ Lenin daran, was er vom ,,modernen [westlichen] Parlamentarismus" hielt, nämlich gar nichts. In allen parlamentarisch regierten Ländern ,,von Amerika bis zur Schweiz“" glichen Parlamente ,Schwatzbuden“. Die eigentlichen „,Staats'geschäfte“ würden ,hinter den Kulissen“ abgewickelt. Den Parlamentarismus der bürgerlichen Gesellschaftsordnung gelte es genauso zu überwinden wie die Herrschaft der Bourgeoisie. Wie bereits Marx befürwortete auch Lenin die Idee, Vertretungskörperschaften zukünftig nach dem Modell der Commune nicht als ,,parlamentarische“, sondern als ,,arbeitende Körperschaften“ einzurichten. ${ }^{43}$ Die Parlamentarier müssten ,selbst arbeiten, selbst ihre Gesetze ausführen [...] [und] selbst unmittelbar vor ihren Wählern die Verantwortung tragen." Wie in der Pariser Kommune sollten die ,gesetzgebende“ und die ,vollziehende Tätigkeit" nicht voneinander getrennt werden. ${ }^{44}$ Ohne Vertretungskörperschaften könne man

40 Lenin 1917/1960, 433. Zu Lenins Demokratiebegriff vgl. auch zusammenfassend: v. Beyme 1966, $1142 f$.

41 Lenin spricht hier nicht vom ,allgemeinen Stimmrecht“ der Bürger, sondern von der ,uneingeschränkten Wählbarkeit" der beamteten Personen.

42 Lenin 1917/1960, 434.

43 Lenin 1917/1960, 436. - Kritik übt Lenin in diesem Zusammenhang auch an der Entwicklung der Sowjets in Russland, die „Helden des modrigen Spießbürgertums [...] [hätten diese] nach dem Vorbild des schäbigsten bürgerlichen Parlamentarismus [...] in bloße Schwatzbuden" verwandelt. Ebd.

44 Lenin 1917/1960, 437. 
sich nämlich ,eine Demokratie nicht denken, auch eine proletarische Demokratie nicht “", so gab sich Lenin überzeugt. ${ }^{45}$

Lenins Vision der zu errichtenden neuen revolutionären politischen Ordnung lässt sich folgendermaßen zusammenfassen: Wie Marx betrachtete er die Pariser Kommune als ein herausragendes Beispiel, wie sich die „Geburt der neuen Gesellschaft aus der alten“ in einem ,naturgeschichtlichen Prozess“ vollziehen könne. ${ }^{46}$ Die Existenz von Vertretungskörperschaften betrachtete Lenin in einer „proletarischen Demokratie“ als ebenso selbstverständlich wie die Wählbarkeit und Absetzbarkeit aller Amtsträger. Wie in der Pariser Kommune sollten in der zukünftigen Gesellschaftsordnung (in Russland) Legislative und Exekutive nicht voneinander getrennt und die Parlamentarier für ihre Entscheidungen und deren Ausführung direkt zur Rechenschaft gezogen werden können. Lenin ging es jedoch nicht darum, die in der Pariser Kommune realisierte bzw. entworfene politische Ordnung nach einem revolutionären Umsturz im Zarenreich einfach zu kopieren. Vielmehr betrachtete er den französischen Fall als eine wichtige Etappe der historischen Entwicklung und als ein Exempel, an dem sich revolutionäre Prozesse analysieren lassen. Wichtig erscheint dabei in unserem Zusammenhang, dass Lenin neben der politischen auch die ökonomische Transformation der Gesellschaft anstrebte, was für sein Verständnis von der Teilhabe der bislang unterdrückten sozialen Klassen an der Macht von elementarer Bedeutung war. Zudem sollte man nicht aus den Augen verlieren, dass Lenin die Schaffung des Kommunismus und des KommuneStaates als ein zukünftiges Projekt erachtete, eine historische Etappe, die sich erst nach einer vorübergehenden „Diktatur des Proletariats“ erreichen ließ.

II.2 In welchem Ausmaß gab es politische Partizipation in der späten Sowjetunion?

In der westlichen Partizipationsforschung der 1970er und 1980er Jahre wurden die hier referierten Schriften von Marx und Lenin immer wieder als Referenz für die Erklärung des politischen Systems der Sowjetunion herangezogen und zitiert. ${ }^{47}$ Ein wichtiger Grund für die Re-Lektüre dieser „Klassiker" war die Beobachtung westlicher Politologen, dass sich seit den 1960er Jahren in der UdSSR eine deutliche Aufwertung der Sowjets

45 Lenin 1917/1960, 437.

46 Lenin 1917/1960, 438.

47 Vgl. u. a. Friedgut 1979, 34-41, Hahn 1988, 58-61. 
als Institutionen der Lokalverwaltung und Transmissionsriemen zwischen der zentralen Regierung und der Bevölkerung vor Ort beobachten ließ. ${ }^{48}$ Da bereits Lenin in seinen Aprilthesen gefordert hatte, Russland in eine „Republik von Arbeiter-, Landarbeiter- und Bauerndeputiertenräten“49 umzuwandeln, umgab die Sowjets noch in den 1960er Jahren eine Aura revolutionärer Legitimität. ${ }^{50}$ Lenin betrachtete die Räte, deren Anfänge bis in die Streikbewegung Ende des 19. Jahrhunderts bzw. bis in die erste russische Revolution von 1905 zurückreichten, als eine Art Nukleus, aus denen die Vertretungskörperschaften der „proletarischen Demokratie“ erwachsen sollten. Obwohl die erste sowjetrussische Verfassung vom Juli 1918 die Einrichtung entsprechender Stadt- und Dorfsowjets vorsah, die zumindest auf dem Papier an die von Marx und Lenin beschworenen Strukturen der Pariser Kommune erinnerten, spielten die Räte de facto in den folgenden Jahren als Organe der politischen Partizipation keine zentrale Rolle. Zum einen scheiterte die Einrichtung entsprechender Strukturen am Widerstand der Bevölkerung oder an den Wirren des Bürgerkriegs. Zum anderen gerieten die indirekt gewählten Räte spätestens ab dem Frühjahr 1919 unter die zentrale Kontrolle der Kommunistischen Partei. ${ }^{51}$

Als die KPdSU im Jahr 1961 die „Diktatur des Proletariats“ jedoch für beendet erklärte und die Sowjetunion zur ,sozialistischen Demokratie des ganzen Volkes" 52 deklarierte, formulierte die sowjetische Führung auch das Ziel, der sowjetische Bürger solle nun noch stärker in die politischen Prozesse ,an der Basis“ einbezogen werden. ,Jeder sowjetische Mensch“, so Nikita Chruščev in seinem Rechenschaftsbericht des Zentralkomitee der KPdSU an den 22. Parteitag, ,muss aktiv an der Verwaltung der gesellschaftlichen Angelegenheiten teilnehmen - das ist unsere Losung, unsere Aufgabe. “53

48 Vgl. Friedgut 1979, 56; Adams 1983, 183.

49 Lenin 1917/1959 (These Nr. 5), 5.

50 Friedgut 1979, 44.

51 Bis 1936 wurden die Sowjets indirekt gewählt. Erst die Verfassung von 1936 brachte die gleiche, geheime und direkte Wahl der Sowjet-Deputierten. Die Wahlberechtigung erhielt ein Sowjetbürger mit Vollendung des 18. Lebensjahrs. Seit 1977 (neue Verfassung) fanden alle zweieinhalb Jahre Wahlen zu den lokalen Sowjets statt. Die Republik-Sowjets und der Oberste Sowjet der UdSSR wurden alle fünf Jahre gewählt. Vgl. Friedgut 1979, 71.

52 Im Parteiprogramm der KPdSU wird der Staat als ,,Volksorganisation der Werktätigen“, als ,Staat des gesamten Volkes (obšcenarodnoe gosudarstvo)“, bzw. als „, Organ, das den Interessen und dem Willen des ganzen Volkes Ausdruck verleiht", bezeichnet. Vgl. Buestrich 1995, 57, FN 22; Hahn 1988, 70; Friedgut 1979, 56.

53 Chruschtschow 1961, 110. 
In der neuen sowjetischen Verfassung aus dem Jahr 1977 wurde unterstrichen, dass sich das politische System des Landes in Richtung der weiteren Entfaltung der sozialistischen Demokratie entwickeln solle. Insbesondere die ,steigende Teilhabe (Beteiligung) der Bürger an der Verwaltung der staatlichen und gesellschaftlichen Belange" wurde hier explizit als Ziel formuliert. ${ }^{54}$

Die westliche Partizipationsforschung nahm diese Entwicklung in der Sowjetunion mit Interesse zur Kenntnis. ${ }^{55}$ Vor allem vom quantitativen Ausmaß politischer „Partizipation“ zeigten sich Politologen diesseits des Eisernen Vorhangs beeindruckt: So arbeiteten Anfang der 1970er Jahre nach offiziellen Angaben - rund 2,2 Mio. Sowjetbürger als Deputierte in den rund 50.000 Vertretungskörperschaften (vom Dorf-Sowjet bis zum Obersten Sowjet der UdSSR). ${ }^{56}$ Dabei wurde auch registriert, dass die Mitgliederzahlen der KPdSU und des Komsomol seit dem Tod Stalins nach offiziellen Angaben - überproportionale Zuwachsraten verzeichneten. So waren die Mitgliederzahlen der KP von 1954/55 bis 1963/64 von 6,9 auf 10,4 Mio. gestiegen, was einem Zuwachs von $51 \%$ entsprach, während die Bevölkerung nur um 16 \% (von 120 auf 140 Mio.) gewachsen war. ${ }^{57}$ Auch die Anzahl der im Komsomol engagierten Bürger war von 1963/64 bis 1972/73 um 41 \% (von 22 auf 31 Mio.) gestiegen, während sich die Bevölkerung in diesem Zeitraum nur um $12 \%$ (von 140 auf 156 Mio.) vergrößert hatte. ${ }^{58}$

Eine Diskussion unter westlichen Politologen drehte sich um die Frage, ob es diese Zahlen rechtfertigten, auch in qualitativer Hinsicht von einem hohen $\mathrm{Ma} \beta$ politischer Partizipation in der Sowjetunion zu sprechen. ${ }^{59}$ Kritisch setzte sich die Partizipationsforschung dabei mit der lange vorherrschenden Forschungsmeinung auseinander, dass es sich bei den Erscheinungsformen politischen Engagements in sozialistischen Staaten um rein zeremonielle, regierungsgeleitete und affirmative Formen der Teilhabe gehandelt habe. ${ }^{60}$ So wurde beispielsweise die gängige Position der Totalitarismus-Forschung auf den Prüfstand gestellt, das sowjetische Re-

54 Zit. nach der englischen Übersetzung bei: Adams 1983, 182. Zur „Brežnev-Verfassung" von 1977 vgl. auch Hahn 1988, 73 und Meissner 1985, 329-334.

55 Vgl. exemplarisch Adams 1983, 178 f.

56 Hough 1976, 8; Friedgut 1979, 71.

57 Zahlen nach: Hough 1976, 8.

58 Hough 1976, 8.

59 Schulz 1981, 3-9; Adams 1983, 179. Schulz weist in diesem Zusammenhang auch auf die legendär hohe „Wahlbeteiligung“ bzw. die „Zustimmungsquoten“ bei sowjetischen Wahlen hin.

60 Vgl. u. a. Adams 1983, 178. 
gime habe politische Partizipation bzw. intermediäre Organisationen nur dazu benutzt, um die Bürger zu „,mobilisieren“ und zu kontrollieren. ${ }^{61}$

Jerry Hough stellte beispielsweise 1976 die provokative Frage, woher die westliche Forschung eigentlich wisse, dass die Beteiligung sowjetischer Bürger in öffentlichen Diskussionen keinerlei Einfluss auf die Ausrichtung der sowjetischen Politik habe. Hough gab zu bedenken, dass die westliche Forschung den verschiedenen Formen der Partizipation in der UdSSR durchaus die funktionale Bedeutung zuschreibe, die Identifikation der Bürger mit dem Regime zu festigen, die Regierung mit feedback-Informationen von der „Basis“ zu versorgen sowie Unmut und Unzufriedenheit freizusetzen. Ob die Teilhabe der sowjetischen Bürger am politischen Prozess neben diesen auch noch andere Funktionen erfülle, war für Hough eine offene Frage. „We do not really know", so Houghs lapidare Antwort auf die Frage, ob die Mitarbeit der sowjetischen Bürger in Partei, Sowjets oder gesellschaftlichen Organisationen einen Einfluss auf die ,große Politik“ (major politics) habe. ${ }^{62}$ Fest stand für ihn nur, dass sich die westliche Forschung viel zu lange von den normativen Annahmen der Totalitarismus-Forschung habe leiten lassen und dass es nun an der Zeit sei, diese Prämissen durch empirische Forschungen zu überprüfen. ${ }^{63}$

Es würde den Rahmen dieses Aufsatzes sprengen, die Entwicklung der westlichen Partizipationsforschung zur UdSSR der 1970er und 1980er Jahre en détail nachzuzeichnen. Stattdessen soll im Folgenden auf die damals gängige Definition des Konzeptes ,politische Partizipation“, die Profilierung ausgewählter Forschungsprojekte und einige exemplarische Forschungsergebnisse eingegangen werden.

Hinsichtlich des Konzeptes politischer Partizipation herrschte unter den Sowjetunion-Forschern weitgehende Einigkeit, dass die gängige, vom westlichen Fall abgeleitete Definition zu eng gefasst sei für komparative Studien, die auch Länder jenseits des Eisernen Vorhangs berücksichtigten. ${ }^{64}$ Konsens bestand auch darin, dass dem Engagement der Menschen in Parteien und Wahlkämpfen bzw. der Beteiligung der Bürger an Wahlen in sozialistischen Gesellschaften nicht die gleiche Bedeutung zukommt wie in westlichen Demokratien. Gleichzeitig müssten auch das Engagement in

61 These von Brzezinski/Huntington 1964, referiert bei Hough 1976, 14. Kritisch zur Totalitarismus-Forschung auch: Schulz 1981, 2; Hahn 1988, 30. Zur Deutung sowjetischer Wahlergebnisse: White 1977, 39.

62 Hough 1976, 19.

63 Hough 1976, 19.

64 Z. B. Friedgut 1979, 14. 
Vertretungskörperschaften wie den Sowjets und gesellschaftlichen Organisationen oder die Formulierung von Eingaben an Behörden, Funktionsträger oder Zeitungen als Formen der politischen Partizipation (sowjetischen Typs) gewertet werden. ${ }^{65}$ Vor diesem Hintergrund definierte Theodore Friedgut „Partizipation“ (1979) beispielsweise als
"involvement of citizens in any activities relating to public affairs, including the expression of ceremonial supports and assistance in the administration of commu- nities or the implementation of regime policies" ${ }^{66}$

Um eine politische Tätigkeit als „Partizipation“ zu werten, müsse diese zum einen gemeinschaftsbezogen (community oriented) sein sowie zweitens unbezahlt und drittens freiwillig erfolgen. ${ }^{67}$ Vergleicht man diese Lesart mit der eingangs zitierten Definition von „Partizipation“ bei RainerOlaf Schultze (2005), so fällt auf, dass westliche Sowjetunion-Forscher der 1970er Jahre bereits Zeichen affirmativer Unterstützung der Regierung (z. B. in Zeremonien) als politische „Teilhabe“ der Bevölkerung werteten, während heute nur solche „Formen politischer Beteiligung“ als Partizipation betrachtet werden, die Bürger unternehmen, ,um politische Entscheidungen direkt oder indirekt zu ihren Gunsten zu beeinflussen. “68

Bei der Operationalisierung ihrer Forschungsfragen griff die westliche Partizipationsforschung vor allem auf Interviews zurück, die entweder in der UdSSR (z. B. mit Delegierten in lokalen Sowjets ${ }^{69}$ ) oder in westlichen Staaten (und Israel) mit Auswanderern aus der Sowjetunion geführt wurden. ${ }^{70}$ Befragungen dieser Art zielten nicht nur darauf ab, mehr darüber

65 DiFranceisco und Gitelman unterscheiden drei Formen politischer Partizipation in der Sowjetunion: a) ,formal-ritualistic participation", b) ,citizen-initiated contacts with official persons and institutions whose task is to represent, check upon and run interference with people“, c) ,contacts over implementation (particularized contacting)“. Vgl. DiFranceisco/Gitelman 1984, 78.

66 Friedgut 1979, 19. Eine ähnliche Definition findet sich bei Hahn 1988, 40.

67 Friedgut 1979, 20. Robert S. Sharlet zufolge, könne man in der vergleichenden Forschung nur dann von Formen politischer Partizipation sprechen, wenn sich diese durch a) Wirksamkeit (efficacy), b) Freiwilligkeit (voluntarism) und c) responsiveness auszeichnen. Da auf Erscheinungsformen politischer „Partizipation“ in kommunistischen Systemen keines dieser drei Kriterien zutrifft, könnten diese auch nicht als Beispiele politischer Teilhabe betrachtet werden. Sharlet 1969, 250, zit. nach: Schulz 1981, 7. Kritisch zu Sharlets Verdikt über Formen der politischen Partizipation in der UdSSR: Adams 1983, 180.

68 Schultze 2005, 675.

69 Friedgut 1979.

70 Friedgut 1979, aufbauend auf älteren Studien wie z. B. Inkeles/Bauer 1959. 
zu erfahren, in welcher Form und wie häufig sich die Interviewpartner in der Vergangenheit politisch engagiert hatten. Untersucht wurde auch, mit welcher Motivation, Intention und Erwartungshaltung dies jeweils geschehen war.

Die Ergebnisse der vergleichenden Partizipationsforschung waren mit Blick auf die UdSSR der 1970er und 1980er Jahre - wen sollte es wundern - alles andere als eindeutig und zum Teil zutiefst widersprüchlich. ${ }^{71}$ Auf der einen Seite fanden sich beispielsweise Forscher wie Richard Little, der zu dem Ergebnis kam, dass sich Bürgern in der UdSSR vielfältige und nachhaltige Möglichkeiten für eine politische Beteiligung (political involvement) böten. ${ }^{72}$ Auf der anderen Seite standen Wissenschaftler wie Robert Sharlet, der es kategorisch ablehnte, von der Existenz (wahrer) ,politischer Partizipation“ in der UdSSR zu sprechen. ${ }^{73}$ Andere Politologen wie Theodore Friedgut konstatierten nüchtern, dass dem Sowjetbürger „, the dimension of citizen initiative" fehle und er in seinem Alltag in erster Linie von ,conformity rather than initiative“ geleitet werde. ${ }^{74}$ Natürlich hing die Antwort auf die Frage, ob es in der UdSSR Formen der politischen Partizipation gab, auch davon ab, welche Definition des Konzepts ,Partizipation“ der entsprechenden Studie jeweils zugrunde lag.

Nur wenige Wissenschaftler gingen in ihrer Diagnose so weit wie Sharlet. Die meisten Autoren billigten dem sowjetischen System durchaus zu, dass es seinen Bürgern Möglichkeiten politischer Partizipation eröffnet. Dabei wurde aber immer wieder unterstrichen, dass sich diese in der UdSSR in Form und Bedeutung deutlich von jener in westlichen Demokratien unterschied. Wayne DiFranceisco und Zvi Gitelman sprachen in diesem Zusammenhang beispielsweise von einer ,verborgenen Partizipation“ (covert participation), die sich als Form der ,Schattenpolitik“ (in Analogie zur „Schattenwirtschaft" ${ }^{\text {") beschreiben lasse. }}{ }^{75}$ Offen war für viele Sowjetologen

71 Eine knappe Zusammenfassung der Forschung bis 1990 findet sich bei: Bahry/Silver 1990, 822f.

72 Little 1976, 446f. hier zit. nach Schulz 1981, 8. Little definiert „Partizipation“ als "the actions, interactions and reactions of citizens with respect to the processes by which the social-economic-political conditions of their lives are established, and the conditions themselves." Referiert bei: Adams 1983, 181.

73 Sharlet 1969, zit. nach: Adams 1983, 180. Zu einem ähnlichen Schluss kommt auch Walter Connor, der nur die sowjetische Dissidentenbewegung als Ausdruck „politischer Partizipation“ in der UdSSR gelten lassen will. Referiert bei Hahn 1988, 32.

74 Friedgut 1979, 302.

75 DiFranceisco/Gitelman 1984. 
die Frage, wie sich das wachsende Maß politischer Partizipation in der UdSSR in Zukunft auf die politische Kultur des Landes auswirken würde. Konsens bestand jedoch darin, dass die entsprechenden Impulse immer „,von oben“ ausgehen würden und auch zunehmende Teilhabe der Bevölkerung am politischen Prozess seit den 1950er Jahren als Produkt und nicht als Quelle eines zu beobachtenden innenpolitischen Wandels anzusehen sei. $^{76}$

Dass die wachsende politische Partizipation der sowjetischen Bevölkerung gar zum Sturz des ganzen Systems beitragen könnte, war für die amerikanischen Politologen in den späten 1970er Jahren noch ein völlig fremder Gedanke. Theodore Friedgut gab sich beispielsweise überzeugt, dass das sowjetische System schon zahlreiche Krisen überstanden habe und auch noch weitere Krisen meistern werde. ${ }^{77}$ Die wachsenden Spielräume politischer Teilhabe, die sich den sowjetischen Bürgern boten, würden, so Friedgut, von der Regierung zur Krisenbewältigung eingesetzt und trügen eher zur Stabilisierung denn zur Erosion des Systems bei. ${ }^{78}$ Auch Jan Adams gab sich 1983 überzeugt, dass Reformen auch in Zukunft ,von oben" angestoßen werden würden. ${ }^{79}$ Allerdings traute er den sowjetischen Bürgern bereits zu, dass diese nur Mittel und Wege finden müssten, um ihre Rechte auf Partizipation voll wahrzunehmen: ,If they succeed, they may indeed transform the system." 80

\section{Fazit: Warum war die Partizipationsforschung blind für den gesellschaftlichen und politischen Wandel?}

Warum entwickelte die westliche Partizipationsforschung, die sich mit der politischen Kultur in der Sowjetunion in den 1970er und 1980er Jahren befasste, kein Gespür für die Legitimationskrise und die Erosionsprozesse innerhalb des UdSSR, die aus heutiger Sicht zum Fall des Regimes beitrugen und die sich rückblickend bereits in den 1970er Jahren abzeich-

76 Friedgut 1979, 289.

77 Selbstkritisch zur politikwissenschaftlichen Osteuropa-Forschung der 1970er und 1980er Jahre: v. Beyme 1994, $16 \mathrm{ff}$.

78 Friedgut 1979, 297.

79 Adams 1983, 188.

80 Adams 1983, 189. 
neten ${ }^{81}$ Zum einen lag dies allem Anschein nach darin begründet, dass sich die westliche Partizipationsforschung lange Zeit vorrangig mit den konventionellen und legalen Formen politischer Partizipation befasste, die das System seinen Bürgern (z. B. im Rahmen der Institutionen der Sowjets oder verschiedener gesellschaftlicher Organisationen) zubilligte. ${ }^{82}$ Unkonventionelle oder illegale Formen der politischen Teilhabe, z. B. im Rahmen der Dissidentenbewegung, des Samizdat, Tamizdat oder Magnitizdat, wurden von der Partizipationsforschung nicht einmal am Rande diskutiert. ${ }^{83}$ Dies erstaunt umso mehr, vor dem Hintergrund, dass dieser Forschungszweig seinen Boom in den späten 1960er und 1970er Jahren nicht zuletzt dem Aufkommen unkonventioneller Formen politischer Partizipation in den westlichen Gesellschaften verdankte und westliche Sowjetologen in diesen Jahren mit dem Aufbau großer Sammlungen sowjetischer Samizdat- und Tamizdat-Literatur begannen. Beim Blick auf die andere Seite des Eisernen Vorhangs blieben die Partizipationsforscher dennoch merkwürdig stark systemfixiert. Dieser Scheuklappenblick hatte weiterhin mit jenen Vorannahmen zu tun, die bei der Analyse des politischen Systems des ,Ostblocks“ zur Anwendung kamen. Zwar traten die Partizipationsforscher mit dem Anspruch an, die normativen Prämissen der TotalitarismusForschung zu überwinden. Die Annahme, dass sich die Gemeinwesen Osteuropas als eine spezifische politische Kultur (mit gemeinsamen ideologischen „Fundamenten“ etc.) beschreiben lässt, wurde dabei jedoch nicht aufgegeben. Bei der Suche nach dem „Wesen“ und dem ideologischen

81 Anfang der 1990er Jahre stellte sich dieser Zusammenhang bereits in einem anderen Licht dar. Vgl. Bahry/Silver 1990. Die Autoren betonen, dass man bei der Analyse politischer Partizipation in der UdSSR stärker als bisher die Werthaltung unterschiedlicher Gruppen berücksichtigen müsse. Der Annahme, die ,,politische Kultur" der Sowjetunion habe die Verhaltensweisen der Menschen in der UdSSR maßgeblich geprägt, wird hier zugunsten einer differenzierten Betrachtung der Gesellschaft mit ihren divergierenden Werthaltungen aufgegeben. Zur Legitimationskrise des sowjetischen Systems in den 1970er Jahren vgl. u. a. Segbers 1989.

82 Eine Ausnahme bildet in diesem Zusammenhang u. a. die Studie von DiFranceisco/Gitelman 1984, die nach der Bedeutung klientelistischer Netzwerke für die Entwicklung ,verdeckter" politischer Partizipation fragten. Darin kommen sie zum ernüchternden Schluss, dass ,the dominant social ethos of Soviet citizens vis-à-vis their government is one of private self interest", und dass die sowjetischen Bürger eine klare Präferenz für „,informal access to and influence on bureaucratic officials“ sowie „, general disdain for formal legalistic procedures and norms" an den Tag legten. Ebd., $618 \mathrm{f}$.

83 Samizdat: Selbstverlag, d. h. Verbreitung illegal vervielfältigter Schriften; Tamizdat: Veröffentlichung verbotener Publikationen im Ausland; Magnitizdat: Vervielfältigung und Verbreitung verbotener Musik auf Tonbändern und Kassetten. 
Fundament dieses Systems gelangten soziale Prozesse, die sich nicht in das vorgegebene Bild fügten, aus dem Blick bzw. gelangten gar nicht erst in den Wahrnehmungshorizont der Betrachter. ${ }^{84}$ Als zusätzlicher Ballast erwiesen sich dabei schließlich ,westliche" theoretische Konzepte, wie jenes der politischen Partizipation. ${ }^{85}$ Diese wurden zwar an die Gegebenheiten der jeweils anderen politischen Kultur angepasst. Dessen ungeachtet trugen sie jedoch nichts oder nur sehr wenig zum Verständnis des politischen Wandels auf der anderen Seite des Eisernen Vorhangs bei, der sich jenseits des Wahrnehmungshorizonts der westlichen Sowjetunionforschung mit weitreichenden historischen Konsequenzen vollzog. Aus dieser Perspektive erscheint auch die historische Auseinandersetzung mit dem Konzept der Partizipation ein lohnendes Unterfangen: Nicht um etwas über die tatsächliche Teilhabe von Menschen an einem politischen Gemeinwesen in der Vergangenheit zu erfahren, sondern über das Problem der Selbstund Fremdwahrnehmung ,,westlicher "Sozialwissenschaftler in der Zeit des Kalten Krieges.

\section{Literaturverzeichnis}

Adams (1983): Jan S. Adams, „Citizen Participation in Community Decisions in the USSR “, in: Peter J. Potichnyj und Jane Shapiro Zacek (Hgg.), Politics and Participation under Communist Rule, New York, 178-195.

Bahry u. Silver (1990): Donna Bahry u. Brian D. Silver, „Soviet Citizen Participation on the Eve of Democratization", The American Political Science Review 84, H. 3, 821-847.

Beissinger (2009): Mark R. Beissinger, „Nationalism and the Collapse of Soviet Communism“, Contemporary European History 18, H. 3, 331-347.

Beyme v. (1966): Klaus von Beyme, „Demokratie“, in: Claus D. Kernig (Hg.), Sowjetsystem und demokratische Gesellschaft: Eine vergleichende Enzyklopädie, Bd. 1, Freiburg/Basel/Wien, 1111-1158.

Beyme v. (1994): Klaus von Beyme, Systemwechsel in Osteuropa, Frankfurt/M.

Brockhaus Enzyklopädie (2006), 21. Auflage, Bd. 21, Leipzig, 65-66, s.v. Partizipation.

Brzezinski u. Huntington (1964): Zbigniew Brzezinski u. Samuel Huntington „Political Power: USA/USSR“, New York.

84 Zur Differenzierung des Bildes einer einheitlichen ,politischen Kultur" in der UdSSR vgl. bereits White 1977, 50-56, wo insbesondere auf die Bedeutung ethnischer Vielfalt innerhalb der Sowjetunion hingewiesen wird.

85 Den ahistorischen Ansatz der „Revisionisten“ kritisierte bereits Cohen 1985, 32. 
Buestrich (1995): Michael Buestrich, Die Verabschiedung eines Systems. Funktionsweise, Krise und Reform der Wirtschaft im Realen Sozialismus am Beispiel der Sowjetunion, Münster.

Buschmann u. Murr (2008): Nikolaus Buschmann u. Karl Borromäus Murr (Hgg.), Treue. Politische Loyalität und militärische Gefolgschaft in der Moderne, Göttingen.

Cohen (1985): Stephen F. Cohen, Rethinking the Soviet Experience. Politics and History since 1917, New York u. Oxford.

Chruschtschow (1961): Nikita Chruschtschow, Rechenschaftsbericht des ZK der KPdSU an den XXII Parteitag vom 17.10.1961, Moskau.

DiFranceisco u. Gitelman (1984): Wayne DiFranceisco u. Zvi Gitelman, „Soviet Political Culture and „Covert Participation“ in Policy Implementation“, The American Political Science Review 78, H. 3, 603-621.

Engels (1878/1953): Friedrich Engels, Herrn Eugen Dührings Umwälzung der Wissenschaft (Anti-Dühring), 6. Auflage, Berlin (Ost).

Fitzpatrick (1992): Sheila Fitzpatrick, „Constructing Stalinism: Reflections on Changing Western and Soviet Perspectives on the Stalin Era“, in: Alec Nove (Hg.), The Stalin Phenomenon, New York, 75-99.

Friedgut (1979): Theodore H. Friedgut, Political Participation in the USSR, Princeton.

Friedrich u. Brzezinski (1956): Carl Joachim Friedrich und Zbigniew Brzezinski, Totalitarian Dictatorship and Autocracy, Cambridge (dt. Totalitäre Diktatur, Stuttgart 1957).

Hahn (1988): Jeffrey W. Hahn, Soviet Grassroots. Citizen Participation in Local Soviet Government, London.

Hildermeier (1997): Manfred Hildermeier, „Interpretationen des Stalinismus“, Historische Zeitschrift 264, 655-674.

Hough (1976): Jerry F. Hough, „Political Participation in the Soviet Union“, Soviet Studies 28, H. 1, 3-20.

Inkeles u. Bauer (1959): Alex Inkeles u. Raymond A. Bauer, The Soviet Citizen, Cambridge.

Kaase (1995): Max Kaase, „Partizipation“, in: Dieter Nohlen (Hg.), Wörterbuch Staat und Politik, München (3. Auflage), 521-527.

Karklins (1994): Rasma Karklins, ,Explaining Regime Change in the Soviet Union“, Europe-Asia Studies 46, Nr. 1, 29-45.

Lenin (1917/1959): Vladimir Il’ič Lenin, „Über die Aufgaben des Proletariats in der gegenwärtigen Revolution (Die Aprilthesen)“, erstmals veröffentlicht in: Pravda, Nr. 26, 7. (20.) April 1917, in: ders., Werke, Bd. 24, Berlin (Ost), $1-8$.

Lenin (1917/1960): Vladimir Il'ič Lenin, „Staat und Revolution. Die Lehre des Marxismus vom Staat und die Aufgaben des Proletariats in der Revolution“, in: ders., Werke, Bd. 25: Juni-September 1917, Berlin (Ost), 393-507. 
Little (1976): D. Richard Little, ,Mass Political Participation in the U.S. and the U.S.S.R.: A Conceptual Analysis“, Comparative Political Studies 8 (1976), H. 4, 437-460.

Manteufel u.a. (2000): Ingo Manteufel, Alexander Steininger u. Jutta Unser (Hgg.), Wohin steuert die Osteuropaforschung? Eine Diskussion, Köln.

Marx (1871/1973): Karl Marx, „Der Bürgerkrieg in Frankreich. Adresse des Generalrats der Internationalen Arbeiterassoziation“, (erstmals in englischer Sprache veröffentlicht, Juni 1871, in deutscher Sprache, Juni/Juli 1871), in: Karl Marx / Friedrich Engels - Werke, Bd. 17, 5. Auflage, Berlin (Ost), 313-365.

Meissner (1985): Boris Meissner, „Die neue Bundesverfassung der UdSSR und die sowjetische Verwaltungsreform von 1973“", in: ders., Partei, Staat und Nation in der Sowjetunion. Ausgewählte Beiträge, Berlin, 305-361.

Mergel (1997): Thomas Mergel, ,Geht es weiterhin voran? Die Modernisierungstheorie auf dem Weg zu einer Theorie der Moderne“", in: ders. u.a. (Hgg.), Geschichte zwischen Kultur und Gesellschaft: Beiträge zur Theoriedebatte, München, 203-232.

Meschkat (1971): Klaus Meschkat, „Pariser Kommune“, in: Claus D. Kernig (Hg.), Sowjetsystem und demokratische Gesellschaft: Eine vergleichende Enzyklopädie, Bd. IV, Freiburg u. a., 1070-1090.

Pateman (1970): Carole Pateman, Participation and Democratic Theory, Cambridge. Plaggenborg (1998): Stefan Plaggenborg, „Die wichtigsten Herangehensweisen an den Stalinismus in der westlichen Forschung“, in: ders. (Hg.), Stalinismus. Neue Forschungen und Konzepte, Berlin, 13-33.

Riescher u. Gabriel (1993): Giesela Riescher und Raimund Gabriel, Die Politikwissenschaft und der Systemwandel in Osteuropa, München.

Rittersporn u. a. (2003): Gábor T. Rittersporn, Jan C. Behrends u. Malte Rolf (Hgg.), Sphären der Öffentlichkeit in Gesellschaften sowjetischen Typs. Zwischen partei-staatlicher Selbstinszenierung und kirchlichen Gegenwelten, Frankfurt/M.

Schulz (1981): Donald E. Schulz, ,Political Participation in Communist Systems: The Conceptual Frontier“, in: ders. u. Jan S. Adams (Hgg.), Political Participation in Communist Systems, New York, 1-25.

Schultze (1995): Rainer-Olaf Schultze, „Partizipation“, in: Dieter Nohlen (Hg.), Lexikon der Politik, Bd. 1: Dieter Nohlen u. Rainer-Olaf Schultze (Hgg.), Politische Theorien, München, 396-406.

Schultze (2005): Rainer-Olaf Schultze, „Partizipation“, in: Dieter Nohlen u. Rainer-Olaf Schultze (Hgg.), Lexikon der Politikwissenschaft, Bd. 2, München (3. Aufl.), 675-677.

Segbers (1989): Klaus Segbers, Der sowjetische Systemwandel, Frankfurt/Main.

Sharlet (1969): Robert S. Sharlet, „Concept Formation in Political Science and Communist Studies: Conceptualizing Political Participation“, in: Frederic J. Fleron (Hg.), Communist Studies and the Social Sciences, Chicago, 244-253.

Simon (1992): Gerhard Simon, „Die Osteuropaforschung, das Ende der Sowjetunion und die neuen Nachfolgestaaten", Aus Politik und Zeitgeschichte, B 52-53, 18.12.1992, 32-38. 
Verba u. a. (1978): Sidney Verba, Norman H. Nie u. Jae-on Kim, Participation and Political Equality. A Seven-Nation Comparison, Cambridge.

Wehler (1975): Hans-Ulrich Wehler, Modernisierungstheorie und Geschichte, Göttingen.

White (1977): Stephen White, „The USSR: Patterns of Autocracy and Industrialism“, in: Archie Brown u. Jack Gray (Hgg.), Political Culture and Political Change in Communist States, London/Basingstoke, 25-65.

White (1979): Stephen White, Political Culture and Soviet Politics, London.

Yurchak (2006): Alexei Yurchak: Everything was Forever, Until it Was No More: The Last Soviet Generation, Princeton.

Zimmermann (2010): Volker Zimmermann u. a. (Hgg.), Loyalitäten im Staatssozialismus: DDR. Tschechoslowakei, Polen, Marburg. 
Brought to you by | Universitaetsbibliothek Basel

Authenticated

Download Date | 11/15/18 12:25 PM 